\section{Automatic image enhancement using intrinsic geometrical information}

\section{H. Soyel and P. W. McOwan}

A new image enhancement algorithm based on nonsubsampled contourlet transform is presented. Low contrast images are transformed into multiscale and multi-directional contour information, where a nonlinear mapping function is used to modify the contour coefficients at each level. The enhancement is achieved by amplifying weak edges and inhibit the background noise while adjusting the dynamic range. Experiments showed that proposed algorithm preserves the intrinsic geometrical information of the enhanced image and can be effectively applied to a wide range of image types under diverse illumination conditions.

Introduction: Conventional Histogram Equalisation (CHE) based histogram modification techniques received the most attention due to their simplicity [1]. Although $\mathrm{CHE}$ and its derivatives can efficiently utilize display intensities, they tend to over enhance the image contrast if there are high peaks in the histogram, often resulting in a harsh and noisy appearance of the output image [2]. One way to approaching this problem is to use multi-scale image decomposition, that is, processing images in each scale independently and recombining each processed image to obtain final image. The advancement of wavelet theory promotes its applications in image contrast entrancement and some promising results are achieved by using multi-scale analysis. The decomposition of images into different frequency ranges, permits the isolation of the frequency components introduced by "intrinsic deformations" or "extrinsic factors" in certain subbands [3]. In [4], singular value based image equalization in discrete wavelet transform domain (DWT-SVE) is proposed to equalize the illumination information in low-low subband image and preserving the high frequency components in the other subbands. However, 2D wavelet transform used is a separable extension of 1D wavelet transform, which does not work well in capturing the geometry of image edges. In this Letter, we propose a new image enhancement method based on nonsubsampled contourlet transform (NSCT) [5]. The proposed algorithm enhances the dynamic range of the image while amplifying weak edges and suppressing noise by modifying the NSCT coefficients using a nonlinear mapping function in each directional subband.

Method: Existing image enhancement algorithms amplify noise when they amplify weak edges since they cannot distinguish noise from weak edges. Since weak edges are geometric structures and noise is not, we can use NSCT to distinguish them. The NSCT is shift-invariant so that each pixel of the transform subbands corresponds to that of the original image in the same spatial location. Therefore, we gather the geometrical information pixel by pixel from the NSCT coefficients. All directional contour subbands can be expressed as

$$
\begin{array}{r}
C_{m, d}, m=1,2, \ldots k, d=1,2, \ldots l_{m} \\
k \in(1,2, \ldots N), l_{m}=2^{N}
\end{array}
$$

where $m$ and $d$ are the scale and direction of the decomposition respectively, $k$ is the number of contour decomposition scale, $l_{m}$ is the number of contour decomposition directions of $m$ th scale and $C_{m, d}$ is the coefficient at the $d$ th directional subband of the $m$ th scale.

The basic idea for image enhancement by multi-scale transforms is to adjust dynamic range while amplifying weak edges and suppressing noise. To this end we modify the NSCT coefficients by a nonlinear mapping function in each scale and direction

$\tilde{C}_{m, d}= \begin{cases}\frac{\max \left(\left|C_{m, d}\right|\right)}{\operatorname{sgn}\left(C_{m, d}\right)} \sin \left(\frac{\pi}{2} \sin \left(\frac{\pi}{2} \frac{\left|C_{m, d}\right|}{\max \left(\left|C_{m, d}\right|\right)}\right)^{\alpha}\right)^{\sqrt{\alpha}}, & m>1 \\ \frac{\max \left(\left|C_{m, d}\right|\right)}{\operatorname{sgn}\left(C_{m, d}\right)} \sin \left(\frac{\pi}{2} \frac{\left|C_{m, d}\right|}{\left.\max \left(\left|C_{m, d}\right|\right)\right)}\right)^{\alpha^{2} / 2}, & m=1\end{cases}$

where

$$
\alpha=\frac{\log \left(\frac{\operatorname{mean}\left(\left|C_{m, d}\right|\right)}{\max \left(\left|C_{m, d}\right|\right)}\right)}{\log \left(\sin \left(\frac{\pi}{2} \frac{\operatorname{mean}\left(\left|C_{m, d}\right|\right)}{\max \left(\left|C_{m, d}\right|\right)}\right)\right)}
$$

This function keeps the coefficients of strong edges, amplifies the coefficients of weak edges and suppresses the noise coefficients in the bandpass directional subbands $(m>1)$ and adjusts the dynamic range in the lowpass subband $(m=1)$. Finally we reconstruct the enhanced image from the enhanced coefficients, $\tilde{C}_{m, d}$, by inverse NSCT.

Results: We compare the enhancement results by the proposed algorithm with those by the CHE and DWT-SVE. In these experiments, for the proposed algorithm we use three scales of decompositions and four directional subbands in each scale. Furthermore, to extend the grey-scale enhancement to colour images, we apply the method to their luminance component only and preserve the chrominance components. Some contrast enhancement results on grey-scale and colour images are shown in Fig. 1. The corresponding mapping functions used are shown in Fig. 2.
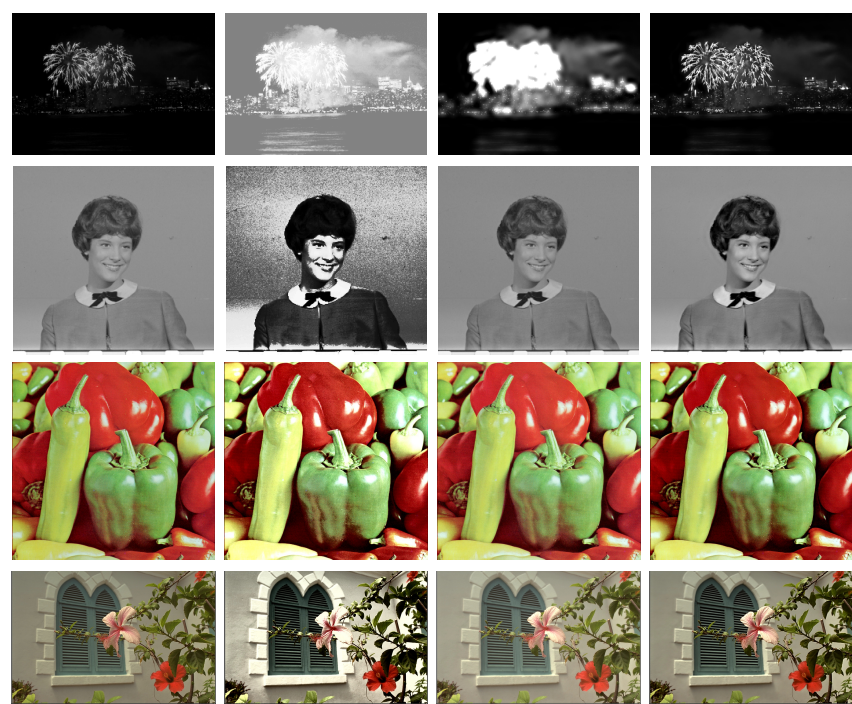

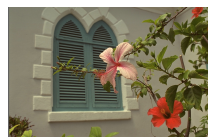

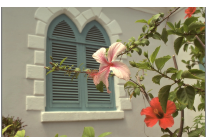

c

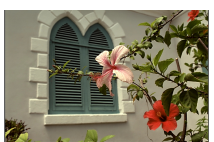

d
Fig. 1 Original and processed versions of 'Fireworks', 'Girl', 'Peppers' and 'Window' images

a Original images

b Enhanced images by CHE

c Enhanced images by DWT-SVE

d Enhanced images by proposed algorithm

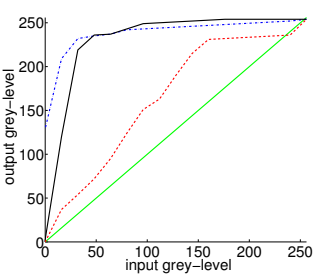

a

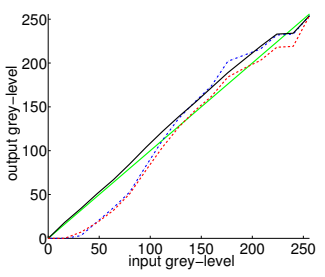

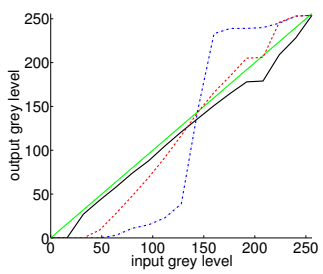

b

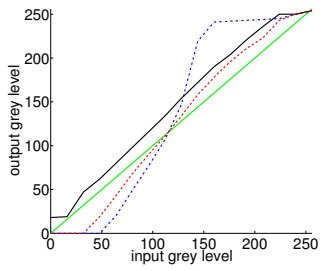

Fig. 2 Mapping functions of enhanced images show in Fig. 1

a Fireworks

b Girl

c Peppers

d Window

key: (green solid line) no change mapping; (blue dash-dotted line) CHE; (black solid line) DWT-SVE; (red dashed line) the proposed algorithm 
The images in Fig. 1 show that the brightness of the input images are enhanced by all the methods but the increase in contrast is not significant and washout effect is apparent for CHE and DWT-SVE. However, in the proposed algorithm, using an adaptive non-linear mapping function in each scale makes it possible to enhance the intensity values of subtle features effectively. Thus, the proposed algorithm improves the overall contrast while preserving image details. This can be seen by comparing mapping functions in Fig. 2, where the grey-levels are excessively stretched or failed to achieve smooth distributions in the other two methods. The proposed algorithm solves this problem by maintaining the background levels in the same interval and is able to adjust the dynamic range and amplify the weak edges. To evaluate the enhancement results objectively, the detailed variance (DV) and background variance (BV) were proposed in [6]. The $\mathrm{DV}$ and $\mathrm{BV}$ values represent the variance of foreground and background pixels,respectively. A good enhancement method should increase the $\mathrm{DV}$ of the original image while $\mathrm{BV}$ value should remain unchanged or, if possible, slightly decrease. We use BV and DV to compare the enhancement performance of CHE, DWT-SVE and the proposed algorithm in Table 1. We observe that our proposed algorithm offers better results in enhancing the image contrast, improving edge sharpness and inhibiting the background noise

Table 1: DV and BV comparisons for enhancement methods

\begin{tabular}{c|c|c|c|c|c|c|c|c}
\hline Image & \multicolumn{2}{|c|}{ Original } & \multicolumn{2}{c|}{ CHE } & \multicolumn{2}{c|}{ DWT-SVE } & \multicolumn{2}{c}{ Proposed } \\
\hline & DV & BV & DV & BV & DV & BV & DV & BV \\
\hline Fireworks & 86.09 & 0.12 & 35.31 & 0.21 & 29.81 & 0.23 & 192.21 & 0.13 \\
\hline Girl & 43.89 & 0.24 & 104.27 & 0.66 & 43.84 & 0.24 & 69.81 & 0.22 \\
\hline Peppers & 20.09 & 0.87 & 35.69 & 0.57 & 20.4 & 0.89 & 30.43 & 0.86 \\
\hline Window & 30.16 & 0.34 & 83.64 & 0.59 & 30.59 & 0.37 & 60.84 & 0.34 \\
\hline
\end{tabular}

Conclusion: We have proposed a novel algorithm for multiscale image enhancement based on NSCT. We have shown that the new algorithm can smooth out the fine textures and noise while simultaneously enhancing the dynamic range and edges in an image. Furthermore, the proposed algorithm can be effectively applied to both grey-level and colour images without any parameter tuning under diverse illumination conditions.

H. Soyel and P. W. McOwan (School of Electronic Engineering and Computer Science, Queen Mary University of London, London, United Kingdom)

E-mail: hsoyel@eecs.qmul.ac.uk

\section{References}

1 Gonzalez, R.C., and Woods, R.E.: 'Digital image processing', (PrenticeHall, 2008)

2 Celik, T., and Tjahjahi T.: 'Automatic image equalization and contrast enhancement using gaussian mixture modeling ', IEEE Trans. Image Process., 2012, 21, (1), pp. 145-156

3 Mallat, S.G.: 'Multifrequency channel decompositions of image and wavelet models', IEEE Trans. Acoust. Speech Signal Process., 1989, 37, (12), pp. 2091-2110

4 Demirel, H., Ozcinar, C., and Anbarjafari, G.: 'Satellite image contrast enhancement using discrete wavelet transform and singular value decomposition', IEEE Geosci. Remote Sens. Lett., 2010, 7, (2), pp. 333-337

5 Cunha da, A.L., Zhou, J.P., and Do, M.N.: 'The nonsubsampled contourlet transform: theory, design, and applications', IEEE Trans. Image Process., 2006, 15, (10), pp. 3089-3101

6 Vanzo, A., Ramponi, G., and Sicaranza, G.L.: 'An image enhancement technique using polynomial filters', Proc. Int. Conf. on Image Processing, 1994, Vol. 2, pp. 477-481 